\title{
Quereinsteiger, Werbeträger, Prominente in der Politik Österreichs
}

\author{
Wolf, Armin: Image-Politik. Prominente Quereinsteiger als Testimonials der Politik, Nomos Ver- \\ lagsgesellschaft, Baden-Baden 2007, 412 Seiten, € 59,--
}

Quereinstiege prominenter Persönlichkeiten in die Politik, seien es Schauspieler, Musiker, Sportler oder Fernsehmoderatoren, treten, so die Prämisse des vorzustellenden Bandes, mit zunehmender Häufigkeit in vielen etablierten Demokratien auf. Der bekannteste „Promi“ und gleichzeitig einer der in seiner politischen Karriere erfolgreichsten dürfte der kalifornische „Gouvernator" Arnold Schwarzenegger sein. Warum aber greifen Parteien auf Prominenz zurück? Wie ergeht es den Prominenten als Politiker? Und wie erfolgreich sind sie in dieser neuen Laufbahn? Das sind die Fragen, die der Band „Image-Politik“ für den österreichischen Fall beantworten möchte.

Der Autor Armin Wolf formuliert seine zentrale Hypothese, wenn auch versteckt, bereits im Untertitel: Er nimmt an, dass prominente Kandidaten vor allem als vertrauenswürdige Werbeträger für die Parteien wirken sollen. Der Begriff des „Testimonials“ - der aus der Werbesprache kommt und leider erst auf Seite 46 in einem Exkurs erläutert wird - bedeutet, dass eine bekannte Persönlichkeit durch ihre Beteiligung an einer Produktwerbung ein „Testimonial“, eine Art Bürgschaft, für das anzupreisende Produkt abgibt. Das Erreichen eines solchen Bürgschaftseffektes unterstellt Wolf als Hauptmotiv für die Anwerbung prominenter Persönlichkeiten durch Parteien.

Als prominenten Quereinsteiger definiert Wolf mit Bezug auf Dietrich Herzog eine überregional bekannte Persönlichkeit, die direkt in eine politische Spitzenfunktion wechselt, ohne vorher Parteikarriere gemacht zu haben (S. 20). Den Gründen für einen Seiteneinstieg sowie der Bewertung seines Erfolges nähert Wolf sich über qualitative Interviews mit den Kandidaten und ihren Rekrutierern sowie durch eine standardisierte Umfrage bei Journalisten und zwei Bevölkerungsumfragen.

In einer ausführlichen Diskussion des Forschungsstandes befasst er sich aber zunächst mit Typologien von Quereinsteigern und dem Begriff der Prominenz in der Mediengesellschaft (S. 25 bis 70). Im Anschluss daran werden theoretische Probleme der politischen Kommunikation (S. 73 bis 81) und generelle Fragen des „politischen Marktes und seiner unberechenbaren Kunden“ (S. 81 bis 133), insbesondere der Wählermotivation und der Mediatisierung von Politik diskutiert. Die Auseinandersetzung mit dem Thema „Politik als Beruf", Professionalisierung von Politik und ihre Folgen, die unter anderem an Jens Borcherts Arbeiten anknüpft, beschließt den konzeptionellen Teil des Bandes (S. 134 bis 190).

Den zentralen Abschnitt bilden die Ausführungen Wolfs zu den 16 untersuchten prominenten Quereinsteigern, die zwischen 1994 und 2002 in Österreich an nationalen und europäischen Wahlen teilgenommen haben. Der hierzulande wohl bekannteste Kandidat dürfte Karl Habsburg sein, der 1996 nach erfolgreicher Wahl für die ÖVP in das Europäische Parlament einzog. In ausführlichen Interviewsequenzen stellt Wolf sowohl die Motive der Quereinsteiger für den Weg in die Politik als auch die Rekrutierungsmotive der Parteien dar. Dabei wird deutlich, dass sich die Parteien tatsächlich vor allem einen Popularitätsund Vertrauenseffekt erhoffen. Dies gilt insbesondere für die FPÖ, für die acht der 16 untersuchten „prominenten Quereinsteiger“ kandidierten.

Der Autor stellt fest, dass prominente Kandidaten in der Regel von der Parteispitze rekrutiert wurden. So war der FPÖ-Vorsitzende Jörg Haider, der für sich in Anspruch nahm, 
dieses Modell für Österreich überhaupt erfunden zu haben, an vier der acht Rekrutierungen seiner Partei direkt beteiligt. Die Kandidaten selbst vermuten in der Regel, angesprochen worden zu sein, um mit ihrem Namen für die Partei zu werben, geben aber selbst meist auch ein substanzielles Interesse an, das sie motiviert habe, sich dieser Herausforderung zu stellen. Die Quereinsteiger sind, das ist Wolfs zweites zentrales Ergebnis, in der Kandidatenrolle wesentlich erfolgreicher als in der darauf folgenden als aktive Politiker. Dass prominente Quereinsteiger deshalb zukünftig aber sogar noch stärker „genutzt“ werden könnten, bezweifelt Wolf. Plausibel erscheint die von Haider vorgetragene Position, dass „es nix nützt, wenn’s alle machen“ (S. 326). Stimmen hinzugewinnen kann in einer Wahl schließlich nur ein Teil der Kandidaten oder Parteien.

Wolfs Buch ist im empirischen Teil lesenswert und aufschlussreich, der theoretische Teil ist jedoch deutlich überdehnt und hätte in dieser Ausführlichkeit in einer Forschungsarbeit nicht publiziert werden müssen. Zur Länge kommen inhaltliche Probleme hinzu: So wird bereits früh das allgemeine Konzept des Quereinsteigers mit dem spezifischen Konzept des prominenten Quereinsteigers vermengt. Ohne darauf bezogene quantitative Analyse wird außerdem der Eindruck vermittelt, dass es sich bei Quereinsteigern grundsätzlich um eher prominentes Personal handele. Später wird diese Aussage implizit wieder zurückgenommen. Die Diskussion Wolfs zum Begriff der politischen Repräsentation orientiert sich normativ deutlich an einem deskriptiven Repräsentationsverständnis. Das reflektiert nicht den Stand der fachlichen Diskussion; vor allem aber kann die Frage nach sozialer Repräsentativität, selbst wenn man sie normativ für berechtigt hält, bei einer Gruppe von 16 Politikern, die in einem Zeitraum von acht Jahren tätig waren, nicht sinnvoll gestellt werden.

Im empirischen Teil werden zudem zentrale Aussagen nicht untermauert. Die auch in der Zusammenfassung auftauchende Feststellung, dass die Quereinsteiger erfolgreiche Kandidaten waren, beruht zum Beispiel nicht auf Analysen, die versuchen, den elektoralen Einfluss dieser Personen zu quantifizieren, sondern auf Einschätzungen der Rekrutierer und der Kandidaten selbst. So „schätzt“ die FPÖ-Kandidatin Liane Höbinger-Lehrer, ihrer Partei „zwei drei Prozent gebracht (zu) haben“ (S. 260). Beide Gruppen aber sind höchst unglaubwürdige Zeugen, wenn es um die Frage geht, wie sehr ihr Einsatz der jeweiligen Partei genützt hat, denn die einen müssen ihre eigene Rolle, die anderen den Rückgriff auf „celebrities" rechtfertigen.

Schließlich muss es als zentraler Mangel angesehen werden, dass die erhebliche Schieflage der Untersuchungsgruppe in Richtung FPÖ - die Hälfte aller untersuchten „Promis“ kandidierten für diese Partei - bei den Auswertungen keine relativierende Bemerkung wert gewesen ist. Vielmehr werden die Ergebnisse so präsentiert, als könnten sie für die rechtspopulistische FPÖ genauso gelten wie beispielsweise für die ÖVP oder die österreichischen Grünen. Das darf jedoch aufgrund der verschiedenen Geschichte, Strukturen und Ziele dieser Parteien bezweifelt werden.

Bei der Menge an präsentiertem Interviewmaterial ärgert außerdem die Form der Darstellung der Interviews. So werden lange Abschnitte mit Interviewzitaten präsentiert, bei denen man lediglich an einer Fußnote bemerkt, dass der Sprecher oder die Sprecherin wechselt. In diesen Fußnoten wiederum werden nur die Namen der Personen geführt, nicht aber die Parteien. Das erschwert dem Nichtösterreicher besonders am Anfang die politische Zuordnung der Kandidaten erheblich. 
Für den Wahl- und Parteienforscher stellt das Buch dennoch eine nützliche Lektüre dar; er kann sich dabei allerdings weitgehend auf den empirischen Teil von „Image-Politik“ beschränken.

Kai-Uwe Schnapp

Schicksale ehemaliger Reichstagsabgeordneter und anderer jüdischer Anwälte nach 1933

Ladwig-Winters, Simone: Anwalt ohne Recht. Das Schicksal jüdischer Rechtsanwälte in Berlin nach 1933, herausgegeben von der Rechtsanwaltskammer Berlin, 2., ergänzte und erweiterte Auflage, be.bra verlag, Berlin 2007, 309 Seiten (205 Abbildungen), € 24,90.

In der Reichshauptstadt lebte „rund ein Drittel aller deutschen Juden“ (S. 13), besonders stark vertreten in der Kaufmannschaft, in den freien und künstlerischen Berufen. Der Anteil der jüdischen Minderheit an der Gesamtbevölkerung Berlins lag 1933 bei 3,8 Prozent. Nach der Ernennung Adolf Hitlers zum Reichskanzler änderte sich die Situation der Juden in Deutschland vollkommen: „Die rassistische Kategorie des ,Juden’ oder ,Nichtariers' (letztere ging über den Kreis der ,Juden' hinaus) wurde alsbald in rechtliche Strukturen umgeformt, die eine andere Realität schufen “(S. 14), vorbereitet von einer Welle des Terrors. So befanden sich unter den mehr als 5.000 nach dem Reichstagsbrand Festgenommenen prominente Rechtsanwälte, unter ihnen der bekannte Strafverteidiger Alfred Apfel, der im August 1933 zusammen mit 13 früheren Reichstagsabgeordneten zu den ersten Ausgebürgerten gehörte: „Volksverräter“ - so die illustrierte Beilage des „Völkischen Beobachters“ „ausgestoßen aus der deutschen Volksgemeinschaft!“

Die Ermordung des SPD-Mitglieds Günther Joachim, der Mandate für die Rote Hilfe übernommen hatte, Ende März 1933 im SA-Gefängnis in der Jüdenstraße konnte noch „als eine Ausnahme“ (S. 80) gelten. Der junge Rechtsanwalt Hans Litten, der 1931 im „Eden-Tanzpalast“-Prozess Hitler im Zeugenstand „äußerst unbequeme Fragen zum Verhältnis der NSDAP zur Gewalt" (S. 27) gestellt hatte, verschwand im Konzentrationslager. 1938 nahm er sich in Dachau das Leben. Kurt Rosenfeld, der sich ebenfalls durch eine Zeugenvernahme Hitlers dessen „persönlichen Hass“ (S. 28) zugezogen hatte, konnte einer Verhaftung durch Flucht in die Tschechoslowakei entkommen. Aber auch „eher unauffällige“ (S. 37) jüdische Anwälte waren schweren Übergriffen ausgesetzt: „Es ist hier kein Hotel Adlon, aber Sie werden es hoffentlich nicht allzu schlecht gehabt haben“(S. 39), gab der SA-Führer bei der Entlassung Fritz Ball höhnisch mit auf den Weg. Ball emigrierte 1939 in die USA. Wie sein Bruder Kurt Ball, der nach Palästina auswanderte und dort 1943/44 „mit der Sammlung von Zeugenaussagen zum Holocaust“ (S. 115) den Grund für die nationale Gedenk- und Forschungsstätte Yad Vashem legte, war er im November 1938 erneut festgenommen und in das KZ Sachsenhausen verbracht worden. Für Justizrat Ernst Ball, der zusammen mit seinen Brüdern praktizierte, konnten nicht einmal die Lebensdaten festgestellt werden. Die Vernichtung wichtiger Dokumente - so hat nur eine von über 1.800 Handakten „die Zeit überstanden“ (S. 12) - zeigt „die Radikalität, mit der die Existenz der jüdischen Anwälte versucht wurde auszulöschen“ (ebenda).

Von den 1933 in Berlin zugelassenen rund 3.400 Anwälten waren 1.835 jüdischer Herkunft, unter ihnen 19 Frauen (S. 58). Die Gesamtzahl ist nicht mehr als ein „Annäherungs- 